\title{
Los estudiantes de medicina como parte del equipo de salud
}

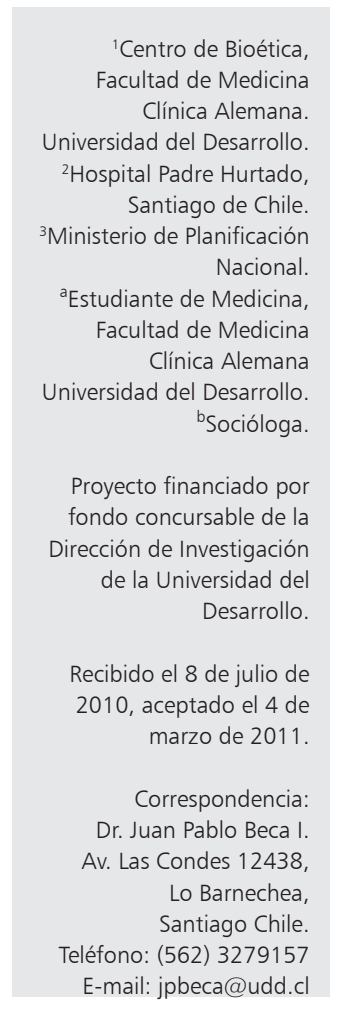

\author{
JUAN PABLO BECA I. ${ }^{1}$, MARÍA INÉS GÓMEZ B. ${ }^{1,2}$, \\ FRANCISCA BROWNE L., ${ }^{3, b}$ JORGE BROWNE S. ${ }^{1, a}$
}

\section{Medical students as members of the health care team}

Background: Teaching hospitals include both undergraduate and postgraduate students, but the role of medical students in the health care team has not been clearly established. Aim: To know the opinion of different professionals about the role of medical students and how this opinion may have an influence in medical education. Material and Methods: A qualitative method was used, asking open questions to focus groups of physicians, nurses and midwives, technicians and undergraduate medical students of $4^{\text {th }}$ and $5^{\text {th }}$ grade. Results: Physicians believe that medical students have no special role in the health care team, nurses think that they may help in communication with patients, and technicians (nurses's aids) value their companionship and closeness with patients. Medical students recognize that their main function is to learn but they are aware that they do help patients. They suggest increasing their integration with other students of other health related careers. Conclusions: Although medical students are usually not seen as part of the health care team, they may fulfill a role with patients during their clinical learning practice. This would improve the quality of their training and the multidisciplinary work of the health care team.

(Rev Med Chile 2011; 139: 462-466).

Key words: Education, medical; Patient care tam; Students, medical.
L

os hospitales que son campos clínicos para la docencia adquieren características y responsabilidades sociales especiales. Una de sus características es que los pacientes atendidos en ellos se relacionan no sólo con los profesionales que los atienden, sino también con docentes y estudiantes de medicina de pre y post-título. La atención de los enfermos la realiza un amplio grupo de personas, profesionales y técnicos paramédicos que se conoce como "equipo de salud". El rol principal de los estudiantes en sus prácticas clínicas es indudablemente su formación profesional, pero no está claro si los estudiantes cumplen alguna función asistencial.

La presencia de los estudiantes de medicina y de otras carreras de la salud en los hospitales docentes se acepta como una responsabilidad institucional, para la cual frecuentemente no se cuenta con las condiciones necesarias. Lo habitual es que los estudiantes sean considerados como observadores externos, más bien ajenos al equipo de salud y sin un rol claro. Sin embargo, dependiendo del nivel en que se encuentran, los estudiantes participan en la atención de pacientes tomando historias clínicas, realizando algunos procedimientos, ayudando en cirugías y evolucionando a los enfermos. Pero es menos clara la participación de los estudiantes en discusiones y decisiones clínicas, o la forma en que los médicos de sala o los tutores reciben sus opiniones, sugerencias o críticas. Más complicadas aun pueden ser algunas situaciones en las cuales ellos son testigos de problemas en la relación clínica o de errores en indicaciones o procedimientos ${ }^{1}$.

Se han estudiado diversos aspectos de la relación de los estudiantes con los pacientes, sobre comunicación y las obligaciones que deben tener con ellos ${ }^{2,3}$. También se ha estudiado la visión de 
Estudiantes de medicina en el equipo de salud - J. P. Beca et al

los enfermos y de los estudiantes sobre su forma de relación ${ }^{4,5}$. Si bien estos estudios permiten fundamentar mejor la enseñanza de actitudes, falta mayor conocimiento acerca del rol de los estudiantes dentro del equipo de salud y la visión que los diferentes estamentos tienen acerca de este rol.

El propósito de este estudio es conocer la opinión de diferentes estamentos y la de estudiantes de $4^{\circ}$ y $5^{\circ}$ año de medicina sobre su rol en el equipo de salud en un hospital docente y analizar su proyección en la formación de los estudiantes.

\section{Método}

Se empleó una metodología cualitativa mediante grupos focales ${ }^{6,7}$. Los temas planteados a los grupos fueron en base a preguntas abiertas de un listado previamente definido (Tabla 1). Las reuniones de los grupos, realizadas en el Hospital Padre Hurtado de Santiago, fueron moderadas por uno de los autores, grabadas y transcritas. Se generó una matriz en una tabla simple en formato

\section{Tabla 1}

1. ¿Cuál es el rol que ustedes cumplen dentro del equipo médico?

2. ¿Están conformes con el rol que actualmente cumplen?

3. ¿Cuál es el rol que el estudiante de medicina debería cumplir dentro del equipo médico?

4. ¿Se encuentran capacitados para desarrollar otras funciones dentro del equipo de salud?

5. ¿Qué otras funciones ustedes podrían asumir dentro del equipo médico?

6. ¿Cuál es el principal aporte que ustedes hacen al equipo médico?

7. ¿Consideran que podrían aportar más de lo que lo hacen dentro del equipo médico?

8. ¿En que grado el actual rol del estudiante de medicina en el equipo de salud es importante para su formación profesional?

9. Según ustedes, ¿Qué opinión tienen los médicos del rol de los estudiantes en el equipo médico?

10. ¿Qué importancia le atribuyen los médicos a la participación de los estudiantes en el equipo médico?

11. ¿Qué importancia le atribuyen los otros profesionales de la salud a la participación de los estudiantes en el equipo médico?
Word de la cual se extrajeron las ideas principales de cada uno de los grupos. La interpretación y el análisis final de los resultados fue realizada por todo el equipo investigador.

Se realizaron 5 grupos focales diferenciados: un grupo de médicos tutores clínicos, un grupo de enfermeras y matronas, un grupo de técnicos paramédicos y dos grupos de estudiantes, uno de $4^{\circ}$ año y uno de $5^{\circ}$ año de medicina. Para cada estamento se invitó a posibles participantes previamente sorteados, asegurando la inclusión de hombres y mujeres. Todos firmaron un documento de consentimiento informado en el cual se explicó el proyecto y se aseguró la confidencialidad de las opiniones. En promedio se contó con 8 participantes por grupo. El proyecto tuvo la aprobación del Comité Institucional de Ética de la Investigación.

\section{Resultados}

Las opiniones de los grupos focales se sintetizaron en sus aspectos esenciales y se presentan separadamente para cada estamento.

\section{Opinión de los médicos tutores}

La opinión general de los médicos fue que los alumnos no cumplen ningún rol útil en el equipo de salud y que significan una carga laboral extra. Sin embargo, reconocen que esto varía según el curso en que se encuentren. Los médicos distinguen entre la situación de alumnos de $4^{\circ}$ y $5^{\circ}$ año de medicina y el internado, período en el cual la relación con el equipo de salud es diferente. También señalan que los alumnos no solamente necesitan conocimientos de medicina, sino también conocer la complejidad de la institución para favorecer su incorporación al equipo. Los médicos no explicitaron posibles aportes de los estudiantes en la atención del enfermo. Sin embargo, reconocen que los obligan a mejorar la práctica clínica y a mantenerse actualizados. También consideran que, aunque infrecuentemente, los estudiantes llevan a modificar conductas médicas, lo cual es considerado como positivo. Finalmente, los docentes evalúan favorablemente a los estudiantes y se sienten comprometidos con el proceso docente.

Las frases siguientes confirman lo anterior: "son un estorbo al trabajo, ... significan una carga laboral ... hay que darse el tiempo de explicar, permitir que un alumno toque un abdomen..." y "cuando hay alumnos también los doctores empiezan 
a hacer las cosas mejor porque tienen más ojos que los miran...".

\section{Opinión de enfermeras y matronas}

Su opinión no fue homogénea, variando mucho según sus especialidades y servicios. Señalan aspectos negativos de los estudiantes de medicina, como ser poco resolutivos o estar poco preparados teórica y socialmente para desempeñarse con pacientes, de tal manera que consideran que su aporte es poco relevante. Además señalaron que no les presentan a los alumnos cuando se incorporan a los servicios y que en algunos casos la relación se dificulta por su prepotencia. Expresaron también que ellas aportan a la formación de los alumnos cuando les enseñan algunos procedimientos y les entregan algunos elementos teóricos. A pesar de lo dicho, las enfermeras consideran útil y positiva la presencia de los estudiantes dentro del servicio, especialmente después de algún tiempo de permanencia. Consideran que los alumnos pueden ser una contribución al hospital en su relación con los pacientes o con sus familiares. Reconocen que los alumnos obligan a las enfermeras a autoexigirse y que se ven obligadas a actualizar sus conocimientos.

Lo anterior se expresa claramente en las frases "la verdad es que no sirven de mucho apoyo ... no son de mucho aporte para la unidad...", y "es una manera de uno exigirse porque también tiene que saber para..."

\section{Opinión de los técnicos}

Para los técnicos el alumno no es un aporte $\mathrm{y}$ les genera trabajo adicional porque cometen errores y no cumplen las normativas. Además sienten que los alumnos se sienten superiores y que en ocasiones tampoco respetan a los pacientes refiriéndose a ellos en forma inadecuada. Sin embargo, expresaron que estas conductas negativas no son mayoritarias pero perjudican al conjunto. También manifiestan que a veces se benefician con enseñanzas de los estudiantes. En relación a las funciones que deberían cumplir los alumnos en el hospital, señalaron que podrían incorporarse más y tener una mayor comunicación con el paciente para explicarles sus diagnósticos y tratamientos. Finalmente, a pesar de algunas apreciaciones negativas iniciales los técnicos consideran que tienen un deber de colaborar con la formación de los estudiantes de medicina.
Las expresiones literales siguientes expresan lo anterior: “... acá no vienen a experimentar con un ratón en una cama, sino a tratar con un paciente que tiene sentimientos...", "se supone que tienen que aprender y en alguna parte tienen que hacerlo, hay que darles las facilidades", y "nos podemos ayudar entre nosotros como un verdadero equipo, como debiera ser".

\section{Opiniones de alumnos de $4^{\circ}$ año de medicina}

Si bien los estudiantes aceptan que no cumplen una función específica, en la práctica asumen un rol de acompañar, escuchar e informar al paciente, lo que mejorara la atención. Además muchas veces aportan información de contexto personal o social de los enfermos, lo que contribuye a tomar mejores decisiones clínicas. Sin embargo, esto varía mucho dependiendo de los distintos servicios y de cada docente. Tienen claro que su rol principal es aprender y que la falta de rol asistencial específico es sólo una etapa en la carrera. Están concientes de que cuando los tutores les piden su opinión se trata de una estrategia docente que les permite aprender a ser parte de un equipo. Sobre su relación con las enfermeras sienten que no son bien acogidos por ellas, contrariamente de lo que ocurre con los técnicos. Finalmente, los alumnos opinan que tanto ellos como el equipo médico se ven beneficiados con su presencia en el hospital porque aprenden aplicando conocimientos teóricos y los médicos se ven obligados a estar al día.

Las siguientes frases ejemplifican lo anterior: "tenemos el rol de apoyar, aconsejar, acoger porque a veces los doctores están apurados y frecuentemente quedan dudas que no se han solucionado", "en un equipo, si falta uno no funciona ... pero si faltamos nosotros funciona todo igual", y "como rol yo creo que el más claro es que tenemos que aprender".

\section{Opiniones de los alumnos de $5^{\circ}$ año de medicina}

Los alumnos de $5^{\circ}$ año también consideran que su rol principal es aprender, haciendo énfasis en la comunicación con el paciente, de tal manera que se ven en un rol de aprendizaje y ayuda al mismo tiempo. Sin embargo, no se sienten parte del equipo, manifestando que depende del docente que ellos se sientan más incorporados, especialmente cuando se discuten casos. Coinciden con los otros grupos en cuanto a que su presencia obliga a los médicos a estar al día y a tener buena disposición 
Estudiantes de medicina en el equipo de salud - J. P. Beca et al

hacia la enseñanza. A diferencia de los alumnos de $4^{\circ}$ año, ellos manifiestan que las enfermeras tienen buena disposición hacia ellos, con lo cual se incorporan más al equipo. Sin embargo, expresan que los estudiantes deben ser formalmente presentados a las enfermeras explicitando su rol en la sala. Otro tema que abordan, para mejorar el sentido del equipo de salud, es la necesidad de tener más interacción con estudiantes de otras carreras de la salud.

Lo anterior se expresa textualmente en frases como "creo que nuestro rol es aprender de ellos, pero también les sirve nuestra participación para reforzar su conocimiento", y "a los pacientes les podemos entregar nuestro tiempo, nuestra buena cara ... pero el equipo de salud tiene que preparase más ..., entonces se está reforzando más y nos está enseñando a nosotros."

\section{Discusión}

Los escasos estudios sobre el tema se refieren más bien a las necesidades de competencias en comunicación ${ }^{8,9}$, a experiencias en trabajo interdisciplinario ${ }^{11,13}$ y a la necesidad de introducir cualidades de profesionalismo en la enseñanza médica desde los primeros años ${ }^{14}$. El presente estudio, con el propósito de conocer el rol que se reconoce a los estudiantes de medicina de medicina en un hospital asistencial-docente no incluyó a los internos porque ellos asumen un rol más claro en el equipo de salud. Además no se estudió este rol en otros campos clínicos, como atención primaria y servicios de urgencia, en donde probablemente puedan existir diferencias en los resultados dado el distinto enfoque en la atención a los pacientes.

Las enfermeras consideraron que el aporte de los estudiantes es mínimo, ya que les dificultan su labor. Otros estudios han destacado la relación difícil que existe entre enfermeras y estudiantes de medicina en hospitales docentes ${ }^{15}$. Los técnicos, aunque no tienen responsabilidad en la formación de los estudiantes, los aceptan positivamente y destacan su rol de acompañamiento de los pacientes.

Los médicos, en su calidad de tutores, son los responsables directos de la formación de los estudiantes en el campo clínico, pero son los que menos valoran su aporte asistencial. Los médicos incorporan bien a los internos en la labor asistencial, pero no tienen claridad acerca del posible rol de los estudiantes de $4^{\circ}$ y $5^{\circ}$ año de medicina en el equipo. No obstante lo anterior, reconocen que los estudiantes les exigen mantenerse al día para responder sus preguntas e inquietudes. La visión de los estudiantes concuerda en algunos aspectos con la de los profesionales y técnicos, específicamente en su rol de aprendices y en los inconvenientes que causan en el trabajo asistencial, pero sugieren que podrían tener un rol más activo. Por otra parte, los estudiantes sugirieron la utilidad de tener en su práctica mayor participación y capacitación multidisciplinar lo que no fue mencionado por otros grupos, pero ha sido señalado en otros estudios ${ }^{13,14,16}$.

El rol fundamental de los estudiantes es de "aprendiz", sin perjuicio de que también pueden ser un aporte al equipo. De las opiniones de los diferentes estamentos es posible inferir una primera descripción en este sentido, como acompañantes de los enfermos, aportando mayor conocimiento acerca de su realidad socio familiar, y colaborando en funciones administrativas. Otros aportes de los estudiantes en el equipo son de tipo indirecto incitando a que los profesionales se mantengan al día, aportando sugerencias y visión crítica, sirviendo como una forma de "control" del trabajo y contribuyendo a la unidad del equipo de salud.

Para comprender mejor el posible rol de los estudiantes en el equipo de salud y favorecer su integración resulta necesario que cada estamento conozca bien su participación y su relación con ellos. Para este efecto se necesita en primer lugar establecer programas de inducción que incluyan rutinas sencillas como presentarlos al equipo antes de iniciar la práctica y explicarles los procedimientos administrativos de la atención de pacientes. A lo anterior hay que agregar la capacitación de los estudiantes para su comunicación con los enfermos y su interacción con los miembros del equipo de salud, junto con una adecuada información a profesionales y técnicos sobre las condiciones y objetivos de la presencia de los estudiantes en los centros asistenciales.

Se ha señalado la imperiosa necesidad de incorporar competencias para el trabajo en equipo como una de los objetivos fundamentales de la educación médica futura ${ }^{17-19}$. Los campos clínicos, variables en su tamaño y complejidad, reciben estudiantes de distintas carreras y pueden constituir una excelente oportunidad para este aprendizaje. Lo anterior, en la medida en que se incorpore a los estudiantes especificando 
su rol y su aporte a los enfermos como acciones complementarias en el concepto de "aprender haciendo". De esta manera, sin afectar la calidad asistencial, se puede lograr una participación del estudiante en el equipo de salud, respetando así las exigencias actuales de acreditación de instituciones asistenciales ${ }^{20}$.

De los resultados obtenidos es posible concluir que, aun cuando en general los estudiantes no son considerados como un aporte a la labor del equipo de salud, ellos pueden asumir un rol que se debe especificar más, para lo cual deben adquirir las competencias respectivas. Esto contribuirá a mejorar la formación de los futuros médicos, el efectivo trabajo multidisciplinar en salud y la calidad de atención a los enfermos. Si bien este estudio tiene las condiciones propias de una investigación cualitativa que no permite generalizar conclusiones, muestra la realidad particular de un hospital público y es posible pensar que ella sea similar en otras instituciones de salud en nuestro país. Se propone que los estudiantes de medicina sean considerados parte del equipo de salud en los hospitales docentes, y que las competencias para trabajar en un equipo multidisciplinario sean incorporadas en la enseñanza médica.

\section{Referencias}

1. Seiden SC, Galvan C, Lamm R. Role of medical students in preventing patient harm and enhancing patient safety. Qual Saf Health Care 2006; 15: 272-6.

2. Beca JP, Browne F, Valdebenito C, Bataszew A, Martínez MJ. Relación Estudiante-Enfermo. Visión del Paciente. Rev Med Chile 2006; 134: 955-9.

3. Beca JP, Browne F, Repetto P, Ortiz A, Salas C. Relación Estudiante de Medicina-Enfermo: La visión de los estudiantes. Rev Med Chile 2007; 135: 1503-9.

4. Anvik T, Gude T, Grimstad H, Baerheim A, Fasmer OB, Hjortdahl P, et al. Assessing medical students' attitudes towards learning communication skills - which components of attitudes do we measure? BMC Med Educ 2007; 7: 4 .

5. García-Huidobro D, Núñez F, Vargas P, Astudillo S, Hitschfeld A, Gennero R, et al. Expectativas de estudiantes de medicina de pregrado en relación al perfil del médico esperado. Rev Med Chile 2006; 134: 947-54.

6. Lincoln IS, Guba EG. Naturalistic Inquiry. Beverly Hills: CA EEUU; 1985. p. 36-43.

7. Kerlinger FN, Lee HB. Investigación del comportamiento: métodos de investigación en ciencias sociales,
México: McGraw-Hill Interamericana Editores. México; 2002.

8. Maudsley G, Williams EM, Taylor DC. Junior medical students' notions of a 'good doctor' and related expectations: a mixed methods study. Med Educ 2007; 41: 476-86.

9. Harward DH, Tresolini CP, Davis WA. Can participation in a health affairs interdisciplinary case conference improve medical students' knowledge and attitudes? Acad Med 2006; 81: 257-61.

10. Nadolski GJ, Bell MA, Brewer BB, Frankel RM, Cushing HE, Brokaw JJ. Evaluating the quality of interaction between medical students and nurses in a large teaching hospital. BMC Med Educ 2006; 6: 23.

11. Miettola J, Mantyselka P, Vaskilampi T. Doctor-patient interaction in Finnish primary health care as perceived by first year medical students. BMC Med Educ 2005; 5 : 34.

12. Page DW. Professionalism and team care in the clinical setting. Clin Anat 2006; 19: 468-72.

13. Gilkey MB, Earp JA. Effective interdisciplinary training: lessons from the University of North Carolina's student health action coalition. Acad Med 2006; 81: 749-58.

14. Young L, Baker P, Waller S, Hodgson L, Moor M. Knowing your allies: medical education and interprofessional exposure. J Interprof Care 2007; 21: 155-63.

15. Nadolski GJ, Bell MA, Brewer BB, Frankel RM, Cushing HE, Brokaw JJ. Evaluating the quality of interaction between medical students and nurses in a large teaching hospital. BMC Medical education 2006; 6: 23-9.

16. Horsburgh M, Lamdin R, Williamson E. Multiprofessional learning: the attitudes of medical, nursing and pharmacy students to share learning. Medical Education 2001; 35: 876-83.

17. Morrison G, Goldfarb S, Lanken PN. Team training of medical students in the $21^{\text {st }}$ century: would Flexner approve?. Acad Med 2010; 85: 254-9.

18. Chacraborti C, Boonyasai RT, Wright SM, Kern DE. A Systematic Review of Teamwork Training Interventions in Medical Student and Resident Education. J Gen Intern Med 2008; 23: 846-53.

19. Hall LW, Scott SD, Cox KR, Gosbee JW, Boshard BJ, Moylan K, et al. Effectiveness of patient safety training in equipping medical students to recognize safety hazards and propose robust interventions. Qual Saf Health Care 2010; 19: 3-8.

20. Manual del Estándar General de Acreditación para Prestadores Institucionales de Atención Cerrada. Ministerio de Salud Chile 2009. Disponible en http://www.redsalud. gov.cl/portal/url/item/65f2651756b874abe0400101 1f011692.pdf 